\title{
Ulrike Worringen: «Patientenschulung ist ein zentraler Behandlungsbaustein in der medizinischen Rehabilitation»
}

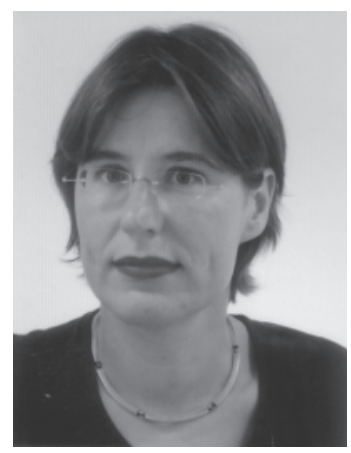

Dr. Ulrike Worringen ist Diplompsychologin und Psychologische Psychotherapeutin (Fachkunde Verhaltenstherapie) und seit 2001 bei der Deutschen Rentenversicherung Bund (ehemals Bundesversicherungsanstalt für Angestellte) tätig, wo sie den Bereich Psychologie und Gesundheitstraining leitet. Sie ist verantwortlich für die Entwicklung von Qualitätsstandards und die fachliche Weiterentwicklung von psychologischen und (psycho)edukativen Behandlungsansätzen in der medizinischen Rehabilitation und hat die Fachaufsicht über die Diplompsychologen in der Reha-Klinikgruppe der Deutschen Rentenversicherung Bund. Die Deutsche Rentenversicherung ist mit rund 800000 Maßnahmen pro Jahr der größte Leistungsund Kostenträger für medizinische Rehabilitation in Deutschland. Die Fragen stellte Prof. Dr. med. Michael Linden, Leiter der Forschungsgruppe Psychosomatische Rehabilitation an der Charité, Campus Benjamin Franklin, Berlin, und der Abteilung Verhaltenstherapie und Psychosomatik des Rehabilitationszentrums Seehof der Deutschen Rentenversicherung Bund, Teltow.

Welche Rolle misst die Deutsche Rentenversicherung als grö $\beta$ ter Träger von medizinischen Rehabilitationsleistungen der Patientenedukation zu?

Ulrike Worringen: Patientenedukation, wir nennen sie Gesundheitstraining oder auch Patientenschulung, ist ein zentraler Behandlungsbaustein der medizinischen Rehabilitation der Deutschen Rentenversicherung. Die Patientenedukation gilt in vielen Indikationen als evidenzbasiert. Sie dient der krankheits- und gesundheitsbezogenen Wissensvermittlung, Einstellungsänderung, Verbesserung von Handlungskompetenz und einer systematischen Nachsorgeplanung.

Die Deutsche Rentenversicherung hat spezielle Materialien für die Patientenedukation zur Verfügung gestellt. Wie sind diese inhaltich und formal aufgebaut?

Ulrike Worringen: Die Deutsche Rentenversicherung hat bereits in den 80er Jahren Schulungskonzepte für die indikationsübergreifenden Themen Ernährung, Bewegung, Stress und Alltagsdrogen entwickelt - die so genannte «Grüne Reihe». Die «Grüne Reihe» wurde in den 90er Jah- ren überarbeitet, aktualisiert und ergänzt durch ressourcenorientierte Ansätze - dem Salutogenesekonzept von Antonovsky folgend. Ergänzend zu dem Thema der Risikofaktoren wurde ein spezifisches Curriculum zum Thema Schutzfaktoren erarbeitet. Dieses neue Programm mit dem Titel «Aktiv Gesundheit fördern» umfasst sowohl primär- als auch sekundärpräventive Aspekte der gesundheitsbezogenen Lebensstiländerung.

Die Deutsche Rentenversicherung Bund hat mit Hilfe ihrer eigenen Reha-Klinikgruppe ein indikationsspezifisches Schulungsprogramm entwickelt, das so genannte Gesundheitstrainingsprogramm. Bei dieser Entwicklung wurde verstärkt die Rehabilitationspraxis miteinbezogen. Mit der Unterstützung von zwei wissenschaftlichen Instituten wurden Schulungsprogramme der eigenen Reha-Klinikgruppe manualisiert und didaktisch aufbereitet. Das Programm umfasst die Indikationsbereiche Kardiologie, Onkologie, Orthopädie, Gastroenterologie, Diabetologie (Typ II), Dermatologie und Pneumologie, ergänzt um Curricula zu den indikationsübergreifenden Themen gesunde Ernährung und Tabakentwöhnung. Beide Programme, das Gesundheitsbildungsprogramm des ehemaligen Verbandes der Deutschen Rentenversicherung

\section{KARGER}

Fax +497614520714

(C) 2006 S. Karger GmbH, Freiburg

E-mail Information@Karger.de www.karger.com/ver

www.karger.com
Dipl.-Psych. Dr. phil. Ulrike Worringen

Deutsche Rentenversicherung Bund, Abt. Rehabilitation Spichernstr. 2

10777 Berlin, Deutschlan

Tel. +49 30 865-58315, Fax -58923

E-mail dr.ulrike.worringen@drv-bund.de 
(VDR) als auch das Gesundheitstrainingsprogramm der Deutschen Rentenversicherung Bund, folgen der Lernzieldidaktik und sind nach einem Baukastenprinzip aufgebaut. Ein Curriculum setzt sich jeweils aus mehreren Modulen (Unterrichtseinheiten) zusammen. Das VDR Gesundheitsbildungsprogramm bietet zudem für jedes Curriculum einen umfangreichen Sachtext. Die didaktische Anleitung ist ebenfalls sehr umfassend. Somit eignet sich dieses Programm auch für Therapeuten, die sich erst als zukünftige Trainer in ein Sachthema und das didaktische Vorgehen der Patientenschulung einarbeiten möchten.

Das indikationsbezogene Gesundheitstrainingsprogramm dagegen setzt das Fachwissen der Trainer voraus und dient primär der Strukturierung der jeweiligen Schulungseinheiten durch eine sehr stringente Aufbereitung der Lerninhalte und Lernziele. Ein umfangreiches Rahmenkonzept klärt über Ziele und Grundlagen des Gesundheitstrainings und das generelle didaktische Vorgehen auf.

Patientenedukation findet vorrangig in Gruppen statt. Welches Gruppenkonzept kommt hier zur Anwendung?

Ulrike Worringen: Geschlossene Gruppen sind didaktisch von Vorteil. Bei einer überschaubaren Anzahl von Teilnehmern (maximal 15) können sich die Patienten im Laufe der Schulung kennen lernen. So kann eine Vertrauenssituation geschaffen werden, die ein offenes Gespräch - auch über belastende krankheitsbezogene Erfahrungen - ermöglicht. Persönliche Einstellungen, die möglicherweise zwingenden krankheitsbezogenen Verhaltensänderungen entgegenstehen, werden unter diesen Umständen von den Patienten eher problematisiert. Um einen möglichst angstfreien Gesprächsraum zu schaffen, bietet es sich an, zunächst vermeintlich «sachliche» Themen in den Vordergrund der Schulung zu stellen. Schulungseinheiten mit emotionalen Inhalten wie das Thema Krankheitsbewältigung sollten erst im weiteren Verlauf der Schulung zum Gegenstand werden. Bedeutsam ist immer die gute interdisziplinäre Zusammenarbeit des Schulungsteams. Es müssen sowohl sachliche Widersprüche vermieden werden als auch vermeintliche «Psychothemen» von Ärzten und anderen nicht psychologischen Trainern ernst genommen und in die eigenen Lerninhalte integriert werden.

Wie unterscheiden sich solche Gruppen von Psychotherapiegruppen im engeren Sinne?

Ulrike Worringen: Emotionale Themen stehen nicht im Zentrum der Gruppenarbeit. Psychische Auffälligkeiten einzelner Rehabilitanden werden gewöhnlich im Rahmen der Patientenschulung nicht thematisiert. Erweisen sich einzelne Rehabilitanden im Rahmen der Schulung als psychisch auffällig, wird ihnen ein individuelles psychologisches Beratungsgespräch angeboten. Psychotherapie im engeren Sinne findet im Rahmen des Gesundheitstrainings nicht statt.
Wer führt solche Gruppen durch und welche Qualifikation müssen Gruppenleiter haben?

Ulrike Worringen: Grundsätzlich werden Patientenschulungen interdisziplinär durchgeführt, die Schulung wird also von mehreren Personen unterschiedlicher Grundqualifikation durchgeführt. In der Regel sind immer ein Arzt und ein Psychologe beteiligt. Abhängig von den weiteren Inhalten der Schulung sind Berufsgruppen wie Diätassistenten, Physiotherapeuten, Stomatherapeuten, Pflegekräfte und Sozialarbeiter eingebunden.

Erwünscht ist eine Fortbildung in Gruppenmoderation. Jedoch sind der Umfang und die Qualität der existierenden Fortbildungsangebote sehr heterogen. Teilweise werden Fortbildungen von Fachgesellschaften und/oder Autoren spezifischer Schulungsprogramme angeboten. Geht die Grunderkrankung mit einer Medikation einher, die eine Mitwirkung der Rehabilitanden erfordert, werden Trainerfortbildungen häufig von der Arzneimittel herstellenden Industrie gefördert.

Psychoedukative Gruppen werden vor allem in Kliniken durchgeführt. Welche Rolle kommt der Einbindung der Patienten in die Gruppe der Mitpatienten während des Aufenthalts zu?

Ulrike Worringen: Psychoedukative Gruppen werden sowohl in stationärem Setting als auch in ambulanten Rehabilitationseinrichtungen angeboten - die Einbindung in die Gruppe der Mitpatienten kann in beiden Settings erfolgen. Die Mitpatienten bilden die «Peergroup» - sie sind in der Regel von derselben oder einer ähnlichen Erkrankung betroffen. Patienten können von Mitpatienten im positiven Sinne lernen, «mit der Erkrankung zu leben». Das Erleben in der Gruppe kann als krankheitsbezogene und auch als allgemeine, soziale Unterstützung erlebt werden. Insbesondere können Rehabilitanden von einem Gruppenansatz profitieren, die in ihrem Alltag eher sozial isoliert leben.

Themen, die in dem Zusammentreffen mit Mitpatienten als belastend erlebt werden, müssen gegebenenfalls therapeutisch aufgegriffen werden. Dies kann bis zu einem gewissen Umfang die Patientenschulung leisten. Belastungen in höherem Ausmaß bedürfen aber gegebenenfalls einer psychologischen bzw. psychotherapeutischen Einzelintervention außerhalb der Gruppenschulung.

Welche Maßnahmen können die Einbindung von Patienten in die Klinik-Gruppe fördern? Wie muss das therapeutische Milieu gestaltet sein, um einen therapeutischen Kontakt von Patienten untereinander zu fördern?

Ulrike Worringen: Grundsätzlich wird die Gruppenbindung erhöht, wenn «emotionale Nähe» und eine gemeinsame «Gruppenidentität» unter den Patienten entsteht. Dies kann durch das Konzept der geschlossenen Gruppe gefördert werden. Rehabilitanden mit ähnlichem Behandlungsbedarf wer- 
den in so genannten «geschlossenen Gruppen» gemeinsam behandelt. Dieses Behandlungskonzept kann über die Patientenschulung im engeren Sinne hinausgehen.

Wir haben grundsätzlich positive Erfahrungen gemacht mit Rehabilitandengruppen, die ihre «Kerntherapie» als Gruppe gemeinsam beginnen und bis zum Abschluss der Rehabilitationsmaßnahme gemeinsam durchführen - etwa täglich ein bis zwei Gruppenveranstaltungen in dieser Gruppenzusammensetzung. Dies führt zu engen Kontakten unter den Gruppenmitgliedern, die auch noch nach Abschluss der Rehabilitation weiterwirken - zum Teil durch weitere persönliche Kontakte oder auch durch selbst initiierte Gruppentreffen. Rehabilitanden, die sonst eher sozial zurückgezogen leben oder auch per se Schwierigkeiten haben, soziale Kontakte aufzunehmen, hilft ein so organisiertes Gruppengeschehen ebenfalls bei der Kontaktaufnahme und gegebenenfalls positivem Erleben von Gemeinschaft.

Voraussetzung für ein erfolgreiches Gruppengelingen sind überschaubare Gruppengrößen mit maximal 15 Teilnehmern. Die Gruppenleiter müssen hinsichtlich der Gruppenführung geschult sein. Hierzu zählt ein Verständnis für gruppendynamische Prozesse und eine gut ausgebildete Problemlösungskompetenz.

Nicht zuletzt muss es Zeit und Raum für persönliche Begegnungen innerhalb und außerhalb des Gruppengeschehens geben. Diese Zeiten und Räume innerhalb der Gruppenarbeit $\mathrm{zu}$ schaffen, ist primär eine didaktische und therapeutische Aufgabe des Gruppenleiters. Zeit und Raum außerhalb der regulären Therapien für ein Miteinander der Rehabilitanden zu schaffen, ist in erster Linie eine organisatorische und strukturelle Herausforderung an die Therapieplanung und die räumliche Ausstattung der Rehabilitationseinrichtung. Auch die Erreichbarkeit weiterer öffentlicher, sozialer Orte außer- halb der Rehabilitationseinrichtung kann für die Gruppenbildung von positiver Bedeutung sein.

Welche Weiterentwicklungen stehen für die Zukunft an?

Ulrike Worringen: Die Qualitätssicherung der Gruppenleiterfortbildung ist der Deutschen Rentenversicherung Bund ein großes Anliegen. Darüber hinaus ergaben sich aus der Begleitstudie zur Implementierung des Gesundheitstrainingsprogramms Hinweise auf den Unterstützungsbedarf etlicher Rehabilitationseinrichtungen.

Rehabilitationseinrichtungen wünschen sich Schulungsmaterialien, die sie bei der Durchführung des Gesundheitstrainings unterstützen, in Form von Folien und umfangreichen Patientenbroschüren. Sie äußern den Bedarf an qualifizierter Schulung für Mitarbeiter, die die Schulung durchführen. Und sie haben den Wunsch, in Bezug auf das Gesundheitstraining Hilfe bei der Durchführung der internen Qualitätssicherung zu bekommen.

Die Deutsche Rentenversicherung Bund erstellt derzeit Patientenbroschüren in Ergänzung zu den indikationsbezogenen Curricula und erarbeitet eine Rahmenkonzeptionen für Gruppenleiterfortbildungen. Eine Weiterentwicklung sind zudem berufsbezogene Schulungsprogramme, die im Rahmen von unterschiedlichen Forschungsprojekten entwickelt werden.

Weitere Serviceleistungen für die Rehabilitationseinrichtungen sind zukünftig auch vom Zentrum Patientenschulung in Würzburg zu erwarten, einer Einrichtung, deren Aufbau derzeit von der Deutschen Rentenversicherung gefördert wird. Wir hoffen, mit diesen Maßnahmen, die Qualität der Patientenschulungen in der medizinischen Rehabilitation weiterhin zu befördern. 